\title{
Local Discontinuous Galerkin Method for the Impact-Induced Wave in a Slender Cylinder Composed of a Non-Convex Elastic Material
}

\author{
Jinfeng Jiang • Yan Xu
}

Received: 6 October 2013 / Revised: 27 November 2013 / Accepted: 12 December 2013 /

Published online: 25 January 2014

(C) School of Mathematical Sciences, University of Science and Technology of China and Springer-Verlag Berlin Heidelberg 2014

\begin{abstract}
In this paper, we present a numerical scheme based on the local discontinuous Galerkin (LDG) method for the wave propagation of phase transition in a slender cylinder by introducing new temporal auxiliary variables. The stability for the LDG scheme is presented. In order to verify the validity of the LDG scheme, we give the errors and accuracy order of a numerical example. Due to the interaction between the dispersion and the material nonlinearity, some interesting wave patterns occur for different pre-strains and impacts, such as the pattern with transformation front and solitary wave and the pattern with rarefaction wave and solitary wave. We also investigate the interaction of the transformation fronts and rarefaction waves, and demonstrate this interesting wave phenomena.
\end{abstract}

Keywords Local discontinuous Galerkin method · Phase transition · Wave pattern · Slender cylinder

Mathematics Subject Classification (2010) $\quad 65 \mathrm{M} 60 \cdot 74 \mathrm{M} 20 \cdot 35 \mathrm{~L} 05$

\section{Introduction}

It is known in the nonlinear elasticity theory that the one-dimensional dynamics of phase transitions in solids can be modeled by the basic field equations [hyperbolicelliptic partial differential equations (PDEs)]. For example, in the stress-induced dynamic of phase transitions in one-dimensional bar, the model is governed by the following system of conservation laws

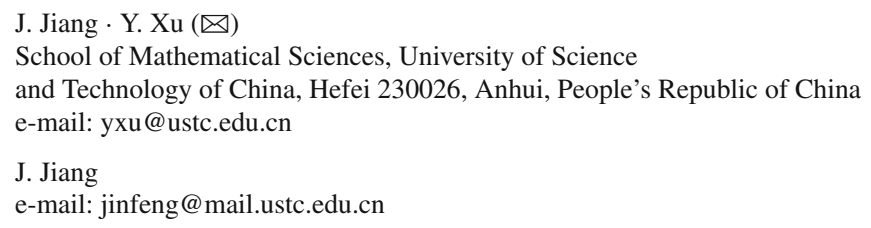


Fig. 1 A diagram of the strain-stress function $\sigma(\gamma) / \mu$

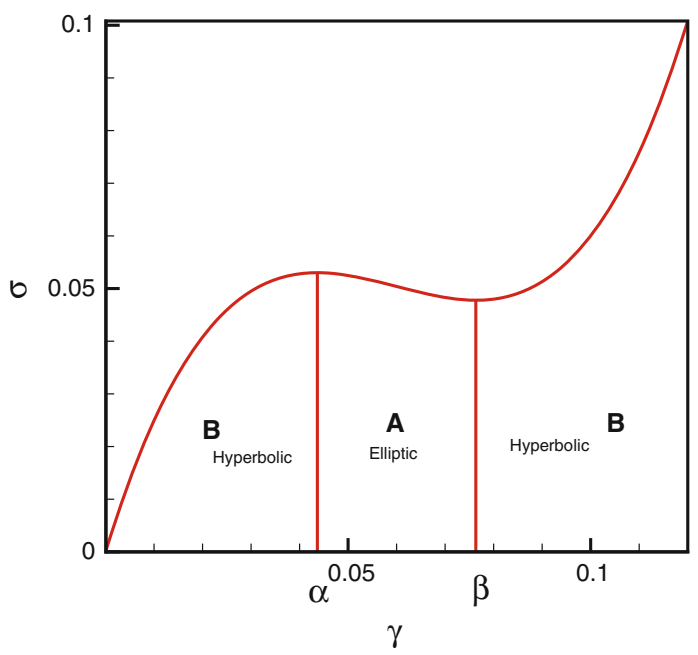

$$
\left\{\begin{array}{l}
\gamma_{t}-u_{x}=0 \\
u_{t}-\rho^{-1} \sigma(\gamma)_{x}=0
\end{array}\right.
$$

where $\gamma, u, \rho$, and $\sigma$ represent the strain, velocity, density, and stress, respectively. If the strain-stress relation $\sigma(\gamma)$ is non-monotonic (see Fig. 1), the system (1.1) is hyperbolic when $\sigma^{\prime}(\gamma)>0$ and elliptic when $\sigma^{\prime}(\gamma)<0$. Then we get a mixed hyperbolic-elliptic system. However, when the solution occurs in the elliptic region, the system is not well-posed. More specifically, the uniqueness of solution is difficult to determine. Fortunately, many authors $[1,2,6,15,17]$ (and the references therein) have carried out studies and analysis to ensure the uniqueness of solution by imposing additional conditions. For example, in [2], Abeyaratne neglected the effects of physical information (e.g., the viscosity, capillarity, and heat conditions) to get the model (1.1). However, Slemrod [15] reconsidered these neglected effects and first proposed the viscosity-capillarity (VC) approach for Riemann solutions to ensure the uniqueness of the solution. Abeyaratne and Knowles [1] introduced a kinetic relation which is an additional condition to conquer the non-uniqueness. Vainchtein [17] considered two dissipation mechanisms (heat conduction and the internal viscous dissipation of kinetic origin) in a finite bar and added a viscoelastic term $\left(\mu u_{x x t}\right.$, where $\mu$ is the viscosity coefficient) for dissipation to the Eq. (1.1) to ensure the uniqueness of the solution. Dai [6] considered the effects of the radial deformation and traction-free condition on the lateral surface when studying a slender circular cylinder, and introduced a dispersive term to ensure the uniqueness of the solution. The experiments in [1013] also suggested that the lateral movement and the radial deformations for phase transitions did play an important part.

For the mixed-type system of conservation laws (1.1), certain numerical schemes have been used to compute the solutions based on the VC approach (see $[3,16]$ and the references therein). Cockburn and Gau [3] implemented a convergence study for different viscosity and capillarity coefficients. Recently, Tian et al. [16] used the local 
discontinuous Galerkin (LDG) method to solve the propagation of phase transition with different viscosity and capillarity coefficients in solids and fluids and presented the error estimate for the LDG scheme. Moreover, Dai and Xu [7], based on the model in Dai [6], made some approximation to the model by omitting the fourth-order terms and obtained a high-order nonlinear equations. Then they applied the fifth-order finite difference weighted essentially non-oscillatory (WENO) scheme to approximate the convective term, and the higher-order central scheme to approximate the high-order and nonlinear terms.

In this paper, we will reinvestigate the model in Dai [6] and obtain a system (see the Eq. (3.1)) by introducing new temporal auxiliary variables rather than by asymptotic approximations in [6]. For this system, we apply the LDG method to carry out numerical simulation and to demonstrate some interesting wave phenomena.

The discontinuous Galerkin (DG) method discussed here is a class of finite element methods. It uses completely discontinuous piecewise polynomial space as the numerical solution space and the test function space in the spatial variables. Using DG method is beneficial for parallel computing and maintaining the high-order accuracy due to this completely local, element-based DG discretization. Additionally, the DG method has the advantage of well hp-adaptation, which consists of local mesh refinement and/or the adjustment of the polynomial order in individual elements. Furthermore, it shares the excellent provable nonlinear stability. The DG method was generalized to the LDG method by Cockburn and Shu [4] to solve the convection-diffusion systems. Likewise, the LDG method enjoys all these excellent properties.

The basic idea of the LDG method is to rewrite equations with higher-order derivatives as a first-order system, and to apply DG method to this system. Many studies on the development of LDG method for the PDEs including the higher-order derivatives have been carried out. For example, Yan and Shu developed the LDG method for $\mathrm{KdV}$-type equations including the third derivatives in [25], and for PDEs including the higher-order derivatives (the fourth and fifth spatial derivatives) in [26]. Xu and Shu further developed the LDG method for a series of nonlinear wave equations in [19-24]. More general information about DG methods as well as their implementation and applications can be found in the review paper by Cockburn and Shu [5].

The structure of this paper is as follows. In Sect. 2, we recall the model problem and derive the dimensionless form of the equations. In Sect. 3, we apply the LDG method to solve the dimensionless form. The time discretization is performed by the third-order total variation diminishing (TVD) Runge-Kutta scheme. The stability for the LDG method is presented in Sect. 4. We present the simulation results in Sect. 5. In order to verify the validity of the LDG method, we give the errors and accuracy. Moreover, we investigate some wave patterns for different pre-strains and different impacts. A summary of the results and the concluding remarks are presented in the final section.

\section{The Model Problem and Its Dimensionless Form}

In this section, we briefly recall the model problem introduced in Dai [6], and derive the dimensionless form of the model problem. 
Dai [6] took into account the effect of other dimensions in a slender circular cylinder with an incompressible phase-transitional material, and established a model equation to model the propagation of phase transitions, which is written in the following form:

$$
\omega_{\tau \tau}-\rho^{-1} \sigma_{x}+\frac{3}{4} a^{2} c_{T}^{2} \omega_{x x x x}-\frac{3}{4} a^{2} \omega_{x x \tau \tau}+\frac{1}{8} a^{2} c_{T}^{-2} \omega_{\tau \tau \tau \tau}=0
$$

where $C_{T}=\sqrt{\mu / \rho}, \mu, \rho$, and $a$ are the shear-wave speed, shear modulus, density, and the radius of the slender cylinder, respectively. In this model, the last three highorder terms can be regarded as dispersive effects under the influence of the radial deformation. These high-order terms can smoothen out a strain discontinuity. Correspondingly, a phase boundary can have a small thickness; we also call such a phase boundary as a transformation front (see [7]).

According to [7], we make the same scaling transformation $t=C_{T} \tau$ and introduce the auxiliary variables

$$
u=\omega_{t}, \gamma=\omega_{x},
$$

and can get

$$
\left\{\begin{array}{l}
\gamma_{t}-u_{x}=0, \\
u_{t}-C_{T}^{-2} \rho^{-1} \sigma_{x}+\frac{3}{4} a^{2} \gamma_{x x x}-\frac{3}{4} a^{2} u_{x x t}+\frac{1}{8} a^{2} u_{t t t}=0,
\end{array}\right.
$$

which is the system (6) in Dai [7]. Here $u$ and $\gamma$ can be regarded as velocity and strain. This system adds three linear dispersive terms to the Eq. (1.1).

In this paper, we assume that the strain-stress function $\sigma(\gamma)$ is non-monotonic; then the system becomes a mixed-type hyperbolic-elliptic system. According to [7], a simple choice is a cubic polynomial written as

$$
\sigma(\gamma)=3 \mu\left(D_{2} \gamma^{3}+D_{1} \gamma^{2}+\gamma\right)
$$

where $D_{1}$ and $D_{2}$ satisfy

$$
D_{1}>0, D_{2}<0 \text { and } 3 D_{2}<D_{1}^{2}<4 D_{2} \text {. }
$$

The case of $D_{1}=-18, D_{2}=100$ is sketched in Fig. 1 .

Further simplifying the Eq. (2.2), we make a scale transformation

$$
\bar{\sigma}=C_{T}^{-2} \rho^{-1} \sigma=\frac{1}{\mu} \sigma,
$$

and obtain $(\bar{\sigma}$ still denoted by $\sigma)$

$$
\begin{aligned}
& \gamma_{t}-u_{x}=0 \\
& u_{t}-\sigma_{x}+\frac{3}{4} a^{2} \gamma_{x x x}-\frac{3}{4} a^{2} u_{x x t}+\frac{1}{8} a^{2} u_{t t t}=0 .
\end{aligned}
$$

In [7], Dai and Xu made further approximation to the system (2.2) by omitting the fourth-order small terms due to the perturbation coefficient $a$ being small and then obtained the following equations: 


$$
\left\{\begin{array}{l}
\gamma_{t}-u_{x}=0 \\
u_{t}-C_{T}^{-2} \rho^{-1} \sigma_{x}+\frac{3}{4} a^{2}\left(\gamma-C_{T}^{-2} \rho^{-1} \sigma\right)_{x x x} \\
+\frac{1}{8} a^{2}\left(C_{T}^{-2} \rho^{-1} \sigma_{\gamma \gamma} u_{x}^{2}+C_{T}^{-4} \rho^{-2} \sigma_{\gamma} \sigma_{x x}\right)_{x}=0 .
\end{array}\right.
$$

Although this system does not include a high-order temporal derivative, it introduces nonlinear terms, increasing the difficulty in space discretization and in stability analysis. For the nonlinear terms, Dai and Xu [7] used a higher-order central scheme to approximate. In this paper, we introduce new temporal auxiliary variables for the Eqs. (2.3a), (2.3b), and use the LDG method to solve (2.3a), (2.3b).

\section{LDG Method and Time Discretization}

In this section, we will present the LDG scheme to solve the high-order equations (2.3a), (2.3b):

$$
\left\{\begin{array}{l}
\gamma_{t}-u_{x}=0 \\
u_{t}-\sigma_{x}+\frac{3}{4} a^{2} \gamma_{x x x}-\frac{3}{4} a^{2} u_{x x t}+\frac{1}{8} a^{2} u_{t t t}=0
\end{array}\right.
$$

\subsection{Notation}

The usual notation of the LDG scheme is adopted. First, we divide the interval $I=$ $\left[x_{L}, x_{R}\right]$ into $N$ cells as follows:

$$
x_{L}=x_{\frac{1}{2}}<x_{\frac{3}{2}}<\cdots<x_{N+\frac{1}{2}}=x_{R} .
$$

We denote

$$
I_{j}=\left(x_{j-\frac{1}{2}}, x_{j+\frac{1}{2}}\right), \quad x_{j}=\frac{1}{2}\left(x_{j-\frac{1}{2}}+x_{j+\frac{1}{2}}\right), \quad j=1, \ldots, N
$$

as the cells and cell center points, respectively. $h_{j}=x_{j+\frac{1}{2}}-x_{j-\frac{1}{2}}$ denotes the length of each cell. We denote $h=\max _{j} h_{j}$ as the length of the largest cell. For simplicity of analysis, we assume that the mesh is regular; that is, the ratio of $h$ over $h_{j}$ for $j=1, \cdots, N$ is upper bounded by a fixed positive constant. We define

$$
V_{h}=\left\{v: v \in P^{k}\left(I_{j}\right), \text { for } x \in I_{j}, j=1, \ldots, N\right\}
$$

as the discontinuous finite element space, where $P^{k}\left(I_{j}\right)$ denotes all polynomials of degree at most $k$ on $I_{j}$. Note that the functions in $V_{h}$ are allowed to be discontinuous across the element interfaces, which is very useful to enhance the numerical behaving. If $k=0$, the considered DG scheme is equivalent to the finite volume scheme. Here and below, $\left(u_{h}\right)_{j+\frac{1}{2}}^{+}=u_{h}\left(x_{j+\frac{1}{2}}^{+}\right)$denotes the right limit of the function $u_{h}$ at the discontinuity point $x_{j+\frac{1}{2}}$, likewise for $u_{h}^{-}$. $\left[u_{h}\right]=u_{h}^{+}-u_{h}^{-}$denotes the jump of $u_{h}$ at each interface point. 


\subsection{The LDG Scheme}

In this subsection, we define our LDG scheme for the Eqs. (2.3a), (2.3b). First, we introduce two temporal auxiliary variables $v, w$, and define

$$
v=u_{t}, \quad w=v_{t} .
$$

We obtain four time evolution equations:

$$
\left\{\begin{array}{l}
\gamma_{t}-u_{x}=0, \\
u_{t}-v=0, \\
v_{t}-w=0, \\
\frac{1}{8} a^{2} w_{t}+v-\sigma_{x}+\frac{3}{4} a^{2} \gamma_{x x x}-\frac{3}{4} a^{2} v_{x x}=0 .
\end{array}\right.
$$

Then we rewrite (3.1) as a first-order system:

$$
\left\{\begin{array}{l}
\gamma_{t}-u_{x}=0, \\
u_{t}-v=0 \\
v_{t}-w=0 \\
\frac{1}{8} a^{2} w_{t}+v-\sigma_{x}+\frac{3}{4} a^{2} p_{x}-\frac{3}{4} a^{2} q_{x}=0,
\end{array}\right.
$$

and

$$
\left\{\begin{array}{l}
p-s_{x}=0 \\
s-\gamma_{x}=0 \\
q-v_{x}=0
\end{array}\right.
$$

where $p, q$, and $s$ are the local auxiliary variables.

Due to the nonlinearity of the function $\sigma(\gamma), \sigma\left(\gamma_{h}\right) \notin V_{h}$. We need to define $\sigma_{h} \in V_{h}$, s.t.

$$
\int_{I_{j}} \sigma_{h} \eta \mathrm{d} x-\int_{I_{j}} \sigma\left(\gamma_{h}\right) \eta \mathrm{d} x=0, \quad \text { for } \forall \eta \in V_{h}
$$

The LDG scheme for (3.2) and (3.3) is formulated as follows: find $u_{h}, \gamma_{h}, v_{h}, w_{h} \in$ $V_{h}$ such that, for all test functions $\phi_{1}, \phi_{2}, \ldots, \phi_{7} \in V_{h}$,

$$
\begin{aligned}
\int_{I_{j}}\left(\gamma_{h}\right)_{t} \phi_{1} \mathrm{~d} x+\int_{I_{j}} u_{h} \phi_{1 x} \mathrm{~d} x-\left(\hat{u}_{h} \phi_{1}^{-}\right)_{j+\frac{1}{2}}+\left(\hat{u}_{h} \phi_{1}^{+}\right)_{j-\frac{1}{2}}=0, \\
\\
\int_{I_{j}}\left(u_{h}\right)_{t} \phi_{2} \mathrm{~d} x-\int_{I_{j}} v_{h} \phi_{2} \mathrm{~d} x=0, \\
\\
\int_{I_{j}}\left(v_{h}\right)_{t} \phi_{3} \mathrm{~d} x-\int_{I_{j}} w_{h} \phi_{3} \mathrm{~d} x=0,
\end{aligned}
$$




$$
\begin{aligned}
& \frac{1}{8} a^{2} \int_{I_{j}}\left(w_{h}\right)_{t} \phi_{4} \mathrm{~d} x+\int_{I_{j}} v_{h} \phi_{4} \mathrm{~d} x+\left(\int_{I_{j}} \sigma_{h} \phi_{4 x} \mathrm{~d} x-\left(\hat{\sigma}_{h} \phi_{4}^{-}\right)_{j+\frac{1}{2}}+\left(\hat{\sigma}_{h} \phi_{4}^{+}\right)_{j-\frac{1}{2}}\right) \\
& \quad-\frac{3}{4} a^{2}\left(\int_{I_{j}} p_{h} \phi_{4 x} \mathrm{~d} x-\left(\hat{p}_{h} \phi_{4}^{-}\right)_{j+\frac{1}{2}}+\left(\hat{p}_{h} \phi_{4}^{+}\right)_{j-\frac{1}{2}}\right) \\
& \quad+\frac{3}{4} a^{2}\left(\int_{I_{j}} q_{h} \phi_{4 x} \mathrm{~d} x-\left(\hat{q}_{h} \phi_{4}^{-}\right)_{j+\frac{1}{2}}+\left(\hat{q}_{h} \phi_{4}^{+}\right)_{j-\frac{1}{2}}\right)=0 \\
& \int_{I_{j}} p_{h} \phi_{5} \mathrm{~d} x+\int_{I_{j}} s_{h} \phi_{5 x} \mathrm{~d} x-\left(\hat{s}_{h} \phi_{5}^{-}\right)_{j+\frac{1}{2}}+\left(\hat{s}_{h} \phi_{5}^{+}\right)_{j-\frac{1}{2}}=0 \\
& \int_{I_{j}} s_{h} \phi_{6} \mathrm{~d} x+\int_{I_{j}} \gamma_{h} \phi_{6 x} \mathrm{~d} x-\left(\hat{\gamma}_{h} \phi_{6}^{-}\right)_{j+\frac{1}{2}}+\left(\hat{\gamma}_{h} \phi_{6}^{+}\right)_{j-\frac{1}{2}}=0 \\
& \int_{I_{j}} q_{h} \phi_{7} \mathrm{~d} x+\int_{I_{j}} v_{h} \phi_{7 x} \mathrm{~d} x-\left(\hat{v}_{h} \phi_{7}^{-}\right)_{j+\frac{1}{2}}+\left(\hat{v}_{h} \phi_{7}^{+}\right)_{j-\frac{1}{2}}=0
\end{aligned}
$$

The "hat" terms in (3.5)-(3.11) at the cell $I_{j}$ boundary due to integration by parts are the so-called "numerical fluxes." To design a successful LDG scheme, it is very important to design the numerical fluxes to ensure the stability of the numerical scheme. The numerical fluxes in (3.5)-(3.11) are chosen as (here we have omitted the index $j+\frac{1}{2}$ of all quantities)

$$
\hat{u}_{h}=u_{h}^{+}, \quad \hat{\sigma}_{h}=\sigma_{h}^{-}, \quad \hat{p}_{h}=p_{h}^{-}, \quad \hat{q}_{h}=q_{h}^{-}, \quad \hat{s}_{h}=s_{h}^{+}, \quad \hat{\gamma}_{h}=\gamma_{h}^{-}, \quad \hat{v}_{h}=v_{h}^{+} .
$$

We remark that the choice of the fluxes (3.12) is not unique; for example, we can also choose the following numerical fluxes

$$
\hat{u}_{h}=u_{h}^{-}, \quad \hat{\sigma}_{h}=\sigma_{h}^{+}, \quad \hat{p}_{h}=p_{h}^{+}, \quad \hat{q}_{h}=q_{h}^{+}, \quad \hat{s}_{h}=s_{h}^{-}, \quad \hat{\gamma}_{h}=\gamma_{h}^{+}, \quad \hat{v}_{h}=v_{h}^{-} .
$$

In fact, in the choice of the numerical fluxes, the crucial part is taking the pair $\hat{u}_{h}$ and $\hat{\sigma}_{h}, \hat{u}_{h}$ and $\hat{p}_{h}, \hat{u}_{h}$ and $\hat{q}_{h}, \hat{u}_{h}$ and $\hat{\gamma}_{h}, \hat{s}_{h}$ and $\hat{\gamma}_{h}, \hat{v}_{h}$ and $\hat{\gamma}_{h}$ from the opposite sides.

In the Sect. 4, we prove that the LDG discretization has a strong stability for both the numerical fluxes (3.12) and (3.13).

Now the definition for the LDG scheme is completed.

\subsection{Time Discretization}

For the spatial discretization discussed above, we will use the time discretization, i.e., the third-order TVD Runge-Kutta method developed by Shu and Osher [14]. For the ordinary differential equations 


$$
\left(U_{h}\right)_{t}=\Gamma\left(U_{h}, t\right)
$$

where $\Gamma$ is a discretization of the spatial operator, the third-order TVD Runge-Kutta method is given by

$$
\left\{\begin{array}{l}
U_{h}^{(1)}=U_{h}^{n}+\Delta t \Gamma\left(U_{h}^{n}, t^{n}\right), \\
U_{h}^{(2)}=\frac{3}{4} U_{h}^{n}+\frac{1}{4} U_{h}^{(1)}+\frac{1}{4} \Delta t \Gamma\left(U_{h}^{(1)}, t^{n}+\Delta t\right), \\
U_{h}^{(n+1)}=\frac{1}{3} U_{h}^{n}+\frac{2}{3} U_{h}^{(2)}+\frac{2}{3} \Delta t \Gamma\left(U_{h}^{(2)}, t^{n}+\frac{1}{2} \Delta t\right) .
\end{array}\right.
$$

\section{The Stability Analysis for LDG Method}

4.1 Stability Analysis for the Eqs. (2.3a), (2.3b)

In this subsection, we take into account the energy of the Eqs. (2.3a), (2.3b) in order to analyze the stability of the LDG scheme. First, we define $W(\gamma)=\int_{\gamma_{0}}^{\gamma} \sigma(\xi) \mathrm{d} \xi$.

Multiplying (2.3a) by $\sigma(\gamma)$ and integrating on $I$, we get

$$
\int_{I} \gamma_{t} \sigma(\gamma) \mathrm{d} x-\int_{I} u_{x} \sigma(\gamma) \mathrm{d} x=0
$$

Similarly, multiplying (2.3b) by $u$ and integrating on $I$, we get

$$
\int_{I} u_{t} u \mathrm{~d} x-\int_{I} \sigma_{x} u \mathrm{~d} x+\frac{3}{4} a^{2} \int_{I} \gamma_{x x x} u \mathrm{~d} x-\frac{3}{4} a^{2} \int_{I} u_{x x t} u \mathrm{~d} x+\frac{1}{8} a^{2} \int_{I} u_{t t t} u \mathrm{~d} x=0 .
$$

Summing up (4.1) and (4.2), and making some operation, we have

$$
\begin{aligned}
& \frac{\mathrm{d}}{\mathrm{d} t} \int_{I}\left(W(\gamma)+\frac{1}{2} u^{2}\right) \mathrm{d} x \\
& =\int_{I} u_{x} \sigma+\sigma_{x} u \mathrm{~d} x-\frac{3}{4} a^{2} \int_{I} \gamma_{x x x} u \mathrm{~d} x+\frac{3}{4} a^{2} \int_{I} u_{x x t} u \mathrm{~d} x-\frac{1}{8} a^{2} \int_{I} u_{t t t} u \mathrm{~d} x \\
& =\left.u \sigma\right|_{I}-\frac{3}{4} a^{2}\left(\left.u \gamma_{x x}\right|_{I}-\left.\gamma_{t} \gamma_{x}\right|_{I}+\frac{\mathrm{d}}{\mathrm{d} t} \int_{I}\left(\frac{1}{2} \gamma_{x}^{2}\right) \mathrm{d} x\right) \\
& \quad+\frac{3}{4} a^{2}\left(\left.u u_{x t}\right|_{I}-\frac{\mathrm{d}}{\mathrm{d} t} \int_{I}\left(\frac{1}{2} u_{x}^{2}\right) \mathrm{d} x\right)-\frac{1}{8} a^{2} \frac{\mathrm{d}}{\mathrm{d} t} \int_{I}\left(\frac{1}{2}\left(u^{2}\right)_{t t}-\frac{3}{2} u_{t}^{2}\right) \mathrm{d} x .
\end{aligned}
$$


Hence,

$$
\begin{aligned}
& \frac{\mathrm{d}}{\mathrm{d} t} \int_{I}\left(W(\gamma)+\frac{1}{2} u^{2}+\frac{3}{4} a^{2}\left(\frac{1}{2} \gamma_{x}^{2}+\frac{1}{2} u_{x}^{2}\right)+\frac{1}{8} a^{2}\left(\frac{1}{2}\left(u^{2}\right)_{t t}-\frac{3}{2} u_{t}^{2}\right)\right) \mathrm{d} x \\
& \quad=\left.\left(u \sigma-\frac{3}{4} a^{2}\left(u \gamma_{x x}-\gamma_{t} \gamma_{x}\right)+\frac{3}{4} a^{2} u u_{x t}\right)\right|_{I} .
\end{aligned}
$$

We define the energy for the Eqs. (2.3a), (2.3b)

$$
E(t)=\int_{I}\left(W(\gamma)+\frac{1}{2} u^{2}+\frac{3}{4} a^{2}\left(\frac{1}{2} \gamma_{x}^{2}+\frac{1}{2} u_{x}^{2}\right)+\frac{1}{8} a^{2}\left(\frac{1}{2}\left(u^{2}\right)_{t t}-\frac{3}{2} u_{t}^{2}\right)\right) \mathrm{d} x
$$

We can see that the effect of the radial deformation is reflected in the energy expression $E(t)$. In fact, the parameter $a \ll 1$; thus, there is a little effect for the energy. But for the existence of these effect, the problem is well-posed.

\subsection{Stability Analysis for the LDG Method}

In this subsection, we would like to investigate the stability of the LDG scheme.

For (3.5) and (3.10), we take the temporal derivative and get

$$
\begin{gathered}
\left.\left.\int_{I_{j}}\left(\gamma_{h}\right)_{t t} \phi_{8} \mathrm{~d} x+\int_{I_{j}}\left(u_{h}\right)_{t} \phi_{8 x} \mathrm{~d} x-\widehat{\left(\left(u_{h}\right)_{t}\right.} \phi_{8}^{-}\right)_{j+\frac{1}{2}}+\widehat{\left(\left(u_{h}\right)_{t}\right.} \phi_{8}^{+}\right)_{j-\frac{1}{2}}=0, \\
\left.\left.\int_{I_{j}}\left(s_{h}\right)_{t t} \phi_{9} \mathrm{~d} x+\int_{I_{j}}\left(\gamma_{h}\right)_{t} \phi_{9 x} \mathrm{~d} x-\widehat{\left(\gamma_{h}\right)_{t}} \phi_{9}^{-}\right)_{j+\frac{1}{2}}+\widehat{\left(\gamma_{h}\right)_{t}} \phi_{9}^{+}\right)_{j-\frac{1}{2}}=0 .
\end{gathered}
$$

Since (4.6), (4.7), and (3.5)-(3.11) hold for any test function in $V_{h}$, we can take the test functions as

$$
\begin{aligned}
& \phi_{1}=\sigma_{h}-\frac{3}{4} a^{2} p_{h}+\frac{3}{4} a^{2} q_{h}, \quad \phi_{2}=u_{h}-\frac{3}{4} a^{2}\left(s_{h}\right)_{t}+\frac{1}{8} a^{2} w_{h}, \quad \phi_{3}=-\frac{1}{8} a^{2} v_{h}, \quad \phi_{4}=u_{h}, \\
& \phi_{5}=\frac{3}{4} a^{2}\left(\gamma_{h}\right)_{t}, \quad \phi_{7}=-\frac{3}{4} a^{2}\left(\gamma_{h}\right)_{t}, \quad \phi_{8}=\frac{3}{4} a^{2}\left(\gamma_{h}\right)_{t}, \quad \phi_{9}=\frac{3}{4} a^{2}\left(s_{h}-\left(u_{h}\right)_{t}-v_{h}\right) .
\end{aligned}
$$

Then summing up the eight Eqs. (4.6), (4.7), (3.5)-(3.9), and (3.11) and using the definition of $\sigma_{h}$ in (3.4) (take $\left.\eta=\left(\gamma_{h}\right)_{t}\right)$, we obtain

$$
\frac{\mathrm{d}}{\mathrm{d} t} \int_{I_{j}}\left(W\left(\gamma_{h}\right)+\frac{1}{2} u_{h}^{2}+\frac{3}{4} a^{2}\left(\frac{1}{2}\left(\gamma_{h}\right)_{t}^{2}+\frac{1}{2} s_{h}^{2}\right)+\frac{1}{8} a^{2}\left(u_{h} w_{h}-\frac{3}{2} v_{h}^{2}\right)\right) \mathrm{d} x
$$




$$
\begin{aligned}
& +\int_{I_{j}}\left(u_{h} \sigma_{h x}+\sigma_{h} u_{h x}\right) \mathrm{d} x-\left(\widehat{u}_{h} \sigma_{h}^{-}\right)_{j+\frac{1}{2}}+\left(\widehat{u}_{h} \sigma_{h}^{+}\right)_{j-\frac{1}{2}}-\left(\widehat{\sigma}_{h} u_{h}^{-}\right)_{j+\frac{1}{2}}+\left(\widehat{\sigma}_{h} u_{h}^{+}\right)_{j-\frac{1}{2}} \\
& -\frac{3}{4} a^{2}\left[\int_{I_{j}}\left(u_{h} p_{h_{x}}+p_{h} u_{h x}\right) \mathrm{d} x-\left(\widehat{u}_{h} p_{h}^{-}\right)_{j+\frac{1}{2}}+\left(\widehat{u}_{h} p_{h}^{+}\right)_{j-\frac{1}{2}}-\left(\widehat{p}_{h} u_{h}^{-}\right)_{j+\frac{1}{2}}\right. \\
& \left.+\left(\widehat{p}_{h} u_{h}^{+}\right)_{j-\frac{1}{2}}\right] \\
& +\frac{3}{4} a^{2}\left[\int_{I_{j}}\left(u_{h} q_{h x}+q_{h} u_{h x}\right) \mathrm{d} x-\left(\widehat{u}_{h} q_{h}^{-}\right)_{j+\frac{1}{2}}+\left(\widehat{u}_{h} q_{h}^{+}\right)_{j-\frac{1}{2}}-\left(\widehat{q}_{h} u_{h}^{-}\right)_{j+\frac{1}{2}}\right. \\
& \left.+\left(\widehat{q}_{h} u_{h}^{+}\right)_{j-\frac{1}{2}}\right] \\
& +\frac{3}{4} a^{2}\left[\int_{I_{j}}\left(\left(\gamma_{h}\right)_{t} s_{h x}+s_{h}\left(\gamma_{h}\right)_{t x}\right) \mathrm{d} x-\widehat{\left(\gamma_{h}\right)_{t}} s_{h}^{-}\right)_{j+\frac{1}{2}} \\
& \left.\left.+\widehat{\left(\gamma_{h}\right)_{t}} s_{h}^{+}\right)_{j-\frac{1}{2}}-\left(\widehat{s}_{h}\left(\gamma_{h}\right)_{t}^{-}\right)_{j+\frac{1}{2}}+\left(\widehat{s}_{h}\left(\gamma_{h}\right)_{t}^{+}\right)_{j-\frac{1}{2}}\right] \\
& +\frac{3}{4} a^{2}\left[\int_{I_{j}}\left(\left(\gamma_{h}\right)_{t}\left(u_{h}\right)_{t x}+u_{h t}\left(\gamma_{h}\right)_{t x}\right) \mathrm{d} x-\widehat{\left(\gamma_{h}\right)_{t}}\left(u_{h}\right)_{t}^{-}\right)_{j+\frac{1}{2}} \\
& \left.\left.\left.\left.+\widehat{\left(\gamma_{h}\right)_{t}}\left(u_{h}\right)_{t}^{+}\right)_{j-\frac{1}{2}}-\widehat{\left(u_{h}\right)_{t}}\left(\gamma_{h}\right)_{t}^{-}\right)_{j+\frac{1}{2}}+\widehat{\left(u_{h}\right)_{t}}\left(\gamma_{h}\right)_{t}^{+}\right)_{j-\frac{1}{2}}\right] \\
& \left.-\frac{3}{4} a^{2}\left[\int_{I_{j}}\left(\left(\gamma_{h}\right)_{t} v_{h x}+v_{h}\left(\gamma_{h}\right)_{t x}\right) \mathrm{d} x-\widehat{\left(\gamma_{h}\right)_{t}} v_{h}^{-}\right)_{j+\frac{1}{2}}+\widehat{\left(\gamma_{h}\right)_{t}} v_{h}^{+}\right)_{j-\frac{1}{2}} \\
& \left.-\left(\widehat{v}_{h}\left(\gamma_{h}\right)_{t}^{-}\right)_{j+\frac{1}{2}}+\left(\widehat{v}_{h}\left(\gamma_{h}\right)_{t}^{+}\right)_{j-\frac{1}{2}}\right]=0 .
\end{aligned}
$$

For convenience, we define two auxiliary functions

$$
F(\alpha, \beta, \widehat{\alpha}, \widehat{\beta})_{j+\frac{1}{2}}=\left(\alpha^{-} \beta^{-}-\widehat{\alpha} \beta^{-}-\alpha^{-\widehat{\beta}}\right)_{j+\frac{1}{2}}
$$

and 
$G(\alpha, \beta, \widehat{\alpha}, \widehat{\beta})_{j-\frac{1}{2}}=\left(\alpha^{-} \beta^{-}-\widehat{\alpha} \beta^{-}-\alpha^{-\widehat{\beta}}-\alpha^{+} \beta^{+}+\widehat{\alpha} \beta^{+}+\alpha^{+} \widehat{\beta}\right)_{j-\frac{1}{2}}$.

Then we have

$$
\begin{aligned}
& \frac{\mathrm{d}}{\mathrm{d} t} \int_{I_{j}}\left(W\left(\gamma_{h}\right)+\frac{1}{2} u_{h}^{2}+\frac{3}{4} a^{2}\left(\frac{1}{2}\left(\gamma_{h}\right)_{t}^{2}+\frac{1}{2} s_{h}^{2}\right)+\frac{1}{8} a^{2}\left(u_{h} w_{h}-\frac{3}{2} v_{h}^{2}\right)\right) \mathrm{d} x \\
& \quad+F\left(u_{h}, \sigma_{h}, \widehat{u}_{h}, \widehat{\sigma}_{h}\right)_{j+\frac{1}{2}}-F\left(u_{h}, \sigma_{h}, \widehat{u}_{h}, \widehat{\sigma}_{h}\right)_{j-\frac{1}{2}}+G\left(u_{h}, \sigma_{h}, \widehat{u}_{h}, \widehat{\sigma}_{h}\right)_{j-\frac{1}{2}} \\
& \quad-\frac{3}{4} a^{2}\left(F\left(u_{h}, p_{h}, \widehat{u}_{h}, \widehat{p}_{h}\right)_{j+\frac{1}{2}}-F\left(u_{h}, p_{h}, \widehat{u}_{h}, \widehat{p}_{h}\right)_{j-\frac{1}{2}}+G\left(u_{h}, p_{h}, \widehat{u}_{h}, \widehat{p}_{h}\right)_{j-\frac{1}{2}}\right) \\
& \quad+\frac{3}{4} a^{2}\left(F\left(u_{h}, q_{h}, \widehat{u}_{h}, \widehat{q}_{h}\right)_{j+\frac{1}{2}}-F\left(u_{h}, q_{h}, \widehat{u}_{h}, \widehat{q}_{h}\right)_{j-\frac{1}{2}}+G\left(u_{h}, q_{h}, \widehat{u}_{h}, \widehat{q}_{h}\right)_{j-\frac{1}{2}}\right) \\
& +\frac{3}{4} a^{2}\left(F\left(s_{h}, \gamma_{h t}, \widehat{s}_{h}, \widehat{\left(\gamma_{h}\right)_{t}}\right)_{j+\frac{1}{2}}-F\left(s_{h}, \gamma_{h t}, \widehat{s}_{h}, \widehat{\left(\gamma_{h}\right)_{t}}\right)_{j-\frac{1}{2}}\right. \\
& \left.+G\left(s_{h}, \gamma_{h t}, \widehat{s}_{h}, \widehat{\left(\gamma_{h}\right)_{t}}\right)_{j-\frac{1}{2}}\right)+\frac{3}{4} a^{2}\left(F\left(u_{h t}, \gamma_{h t}, \widehat{\left(u_{h}\right)_{t}}, \widehat{\left(\gamma_{h}\right)_{t}}\right)_{j+\frac{1}{2}}\right. \\
& \left.\quad-F\left(u_{h t}, \gamma_{h t}, \widehat{\left(u_{h}\right)_{t}}, \widehat{\left(\gamma_{h}\right)_{t}}\right)_{j-\frac{1}{2}}+G\left(u_{h t}, \gamma_{h t}, \widehat{\left(u_{h}\right)_{t}}, \widehat{\left(\gamma_{h}\right)_{t}}\right)_{j-\frac{1}{2}}\right) \\
& \quad-\frac{3}{4} a^{2}\left(F\left(v_{h}, \gamma_{h t}, \widehat{v}_{h}, \widehat{\left(\gamma_{h}\right)_{t}}\right)_{j+\frac{1}{2}}-F\left(v_{h}, \gamma_{h t}, \widehat{v}_{h}, \widehat{\left(\gamma_{h}\right)_{t}}\right)_{j-\frac{1}{2}}\right. \\
& +G\left(v_{h}, \gamma_{h t}, \widehat{v}_{h}, \widehat{\left.\left(\gamma_{h}\right)_{t}\right)_{j-\frac{1}{2}}}\right)=0 .
\end{aligned}
$$

By the definition (3.12) or (3.13) of the numerical fluxes, it is very natural that $\widehat{\left(\gamma_{h}\right)_{t}}=\gamma_{h t}^{-},{\widehat{\left(u_{h}\right)_{t}}}=u_{h t}^{+}$or ${\widehat{\left(\gamma_{h}\right)_{t}}}=\gamma_{h t}^{+}, \widehat{\left(u_{h}\right)_{t}}=u_{h t}^{-}$. Then after some algebraic manipulation, we obtain

$G(\alpha, \beta, \widehat{\alpha}, \widehat{\beta})_{j-\frac{1}{2}}=\left(\alpha^{-} \beta^{-}-\widehat{\alpha} \beta^{-}-\alpha^{-} \widehat{\beta}-\alpha^{+} \beta^{+}+\widehat{\alpha} \beta^{+}+\alpha^{+} \widehat{\beta}\right)_{j-\frac{1}{2}}=0$, where $(\alpha, \beta)=\left(u_{h}, \sigma_{h}\right),\left(u_{h}, p_{h}\right),\left(u_{h}, q_{h}\right),\left(\left(u_{h}\right)_{t},\left(\gamma_{h}\right)_{t}\right),\left(s_{h},\left(\gamma_{h}\right)_{t}\right),\left(v_{h},\left(\gamma_{h}\right)_{t}\right)$.

Summing up the Eq. (4.9) on $j$, we easily obtain

$$
\begin{aligned}
& \frac{\mathrm{d}}{\mathrm{d} t} \int_{I}\left(W\left(\gamma_{h}\right)+\frac{1}{2} u_{h}^{2}+\frac{3}{4} a^{2}\left(\frac{1}{2}\left(\gamma_{h}\right)_{t}^{2}+\frac{1}{2} s_{h}^{2}\right)+\frac{1}{8} a^{2}\left(u_{h} w_{h}-\frac{3}{2} v_{h}^{2}\right)\right) \mathrm{d} x \\
= & -\left(F\left(u_{h}, \sigma_{h}, \widehat{u}_{h}, \widehat{\sigma}_{h}\right)_{N+\frac{1}{2}}-F\left(u_{h}, \sigma_{h}, \widehat{u}_{h}, \widehat{\sigma}_{h}\right)_{\frac{1}{2}}\right) \\
& +\frac{3}{4} a^{2}\left(F\left(u_{h}, p_{h}, \widehat{u}_{h}, \widehat{p}_{h}\right)_{N+\frac{1}{2}}-F\left(u_{h}, p_{h}, \widehat{u}_{h}, \widehat{p}_{h}\right)_{\frac{1}{2}}\right) \\
& -\frac{3}{4} a^{2}\left(F\left(u_{h}, q_{h}, \widehat{u}_{h}, \widehat{q}_{h}\right)_{N+\frac{1}{2}}-F\left(u_{h}, q_{h}, \widehat{u}_{h}, \widehat{q}_{h}\right)_{\frac{1}{2}}\right) \\
& -\frac{3}{4} a^{2}\left(F\left(s_{h}, \gamma_{h t}, \widehat{s}_{h}, \widehat{\left(\gamma_{h}\right)_{t}}\right)_{N+\frac{1}{2}}-F\left(s_{h}, \gamma_{h t}, \widehat{s}_{h}, \widehat{\left(\gamma_{h}\right)_{t}}\right)_{\frac{1}{2}}\right) \\
& -\frac{3}{4} a^{2}\left(F\left(u_{h t}, \gamma_{h t}, \widehat{\left(u_{h}\right)_{t}}, \widehat{\left(\gamma_{h}\right)_{t}}\right)_{N+\frac{1}{2}}-F\left(u_{h t}, \gamma_{h t}, \widehat{\left(u_{h}\right)_{t}}, \widehat{\left(\gamma_{h}\right)_{t}}\right)_{\frac{1}{2}}\right) \\
& +\frac{3}{4} a^{2}\left(F\left(v_{h}, \gamma_{h t}, \widehat{v}_{h}, \widehat{\left(\gamma_{h}\right)_{t}}\right)_{N+\frac{1}{2}}-F\left(v_{h}, \gamma_{h t}, \widehat{v}_{h}, \widehat{\left(\gamma_{h}\right)_{t}}\right)_{\frac{1}{2}}\right) .
\end{aligned}
$$


When the numerical fluxes are chosen as (3.12) or (3.13), the right-hand side of (4.10) agrees with the right-hand side of (4.4). If the boundary conditions are periodic or compact support, then we have

$$
\frac{\mathrm{d}}{\mathrm{d} t} \int_{I}\left(W\left(\gamma_{h}\right)+\frac{1}{2} u_{h}^{2}+\frac{3}{4} a^{2}\left(\frac{1}{2}\left(\gamma_{h}\right)_{t}^{2}+\frac{1}{2} s_{h}^{2}\right)+\frac{1}{8} a^{2}\left(u_{h} w_{h}-\frac{3}{2} v_{h}^{2}\right)\right) \mathrm{d} x=0
$$

\section{Numerical Experiments}

In this section, we will use the LDG scheme combined with the third-order explicit TVD Runge-Kutta method (3.14) to simulate the Eqs. (2.3a), (2.3b), and present numerical examples to illustrate the accuracy and capability of the LDG method. Meanwhile, we will give the initial-boundary-value problem to present some interesting wave patterns.

Example 1 Accuracy test for the Eqs. (2.3a), (2.3b).

According to Dai [7], the two-phase solutions for the Eqs. (2.3a), (2.3b) are written in the following form:

$$
\left\{\begin{array}{l}
\gamma=\frac{\gamma^{+}+\gamma^{-}}{2}+\frac{\gamma^{+}-\gamma^{-}}{2} \tanh \left(-\frac{\gamma^{+}-\gamma^{-}}{a} \sqrt{\frac{3 D_{2}}{6-6 \dot{s}^{2}+\dot{s}^{4}}}(x-\dot{s} t)\right), \\
u=-\dot{s} \gamma+\dot{s} \gamma^{-}-V
\end{array}\right.
$$

where $V$ is determined by the Rankine-Hugoniot jump condition $\left(\sigma^{+}-\sigma^{-}\right)+\dot{s}\left(u^{+}+\right.$ $V$ ) $=0$, (here we let $u^{+}=0$ ), and the traveling velocity $\dot{s}$ is

$$
\sqrt{3 D_{2} \gamma^{+2}+2 D_{1} \gamma^{+}+\frac{9 D_{2}-2 D_{1}^{2}}{3 D_{2}}}
$$

where $\gamma^{+}+\gamma^{-}=-\frac{2 D_{1}}{3 D_{2}}$. Note that (5.1) are not the exact solutions to the system (2.4).

We take the parameters as $a=0.001, V=0.05$; therefore, we have $\gamma^{+}=$ $0.0291987, \gamma^{-}=0.0908013$, and $\dot{s}=0.211225$.

We compute the numerical simulation for the initial two-phase wave in the interval $I=[-0.05,0.15]$ till the final time $t=0.1$. Nature boundary conditions: $u_{x}=$ $0, \gamma_{x}=0, v_{x}=0, w_{x}=0$ at the left boundary are used. We give the $L^{1}$ and $L^{\infty}$ error and the numerical orders of accuracy for strain $\gamma$ with $P^{k}$ element when $k=0,1,2,3$ and the meshes are uniform, see Table 1 .

From Table 1, we can find that the method with $p^{k}$ elements gives a uniform $(k+1)$ th order of accuracy for $\gamma$ in both the $L^{1}$ and $L^{\infty}$ norms. The strain $\gamma$ at $t=0.4$ and $t=0.6$ when $n=128,256$ are shown in Fig. 2 .

Example 2 Different pre-strains and different impacts. 
Table 1 Accuracy test for solution $\gamma$ to $(2.3 \mathrm{a}),(2.3 \mathrm{~b})$, $c f l=0.005, \Delta t=$ cfl $\Delta x^{3} / a^{2}, t=0.1$

\begin{tabular}{|c|c|c|c|c|c|}
\hline$p^{k}$ & $n$ & $L^{1}$ error & Order & $L^{\infty}$ error & Order \\
\hline \multirow[t]{7}{*}{$p^{0}$} & 32 & $6.72 \mathrm{E}-04$ & - & $4.15 \mathrm{E}-02$ & - \\
\hline & 64 & $4.03 \mathrm{E}-04$ & 0.74 & $1.89 \mathrm{E}-03$ & 1.13 \\
\hline & 128 & $3.27 \mathrm{E}-05$ & 3.62 & $2.72 \mathrm{E}-03$ & 2.79 \\
\hline & 256 & $1.31 \mathrm{E}-05$ & 1.32 & $7.47 \mathrm{E}-04$ & 1.87 \\
\hline & 512 & $6.62 \mathrm{E}-06$ & 0.99 & $2.65 \mathrm{E}-04$ & 1.50 \\
\hline & 1,024 & $3.29 \mathrm{E}-06$ & 1.01 & $1.25 \mathrm{E}-04$ & 1.08 \\
\hline & 2,048 & $1.64 \mathrm{E}-06$ & 1.01 & $6.24 \mathrm{E}-05$ & 1.00 \\
\hline \multirow[t]{7}{*}{$p^{1}$} & 32 & $4.57 \mathrm{E}-04$ & - & $3.20 \mathrm{E}-02$ & - \\
\hline & 64 & $1.68 \mathrm{E}-04$ & 1.44 & $9.79 \mathrm{E}-03$ & 1.71 \\
\hline & 128 & $1.78 \mathrm{E}-05$ & 3.24 & $1.95 \mathrm{E}-03$ & 2.33 \\
\hline & 256 & $2.34 \mathrm{E}-06$ & 2.92 & $3.06 \mathrm{E}-04$ & 2.67 \\
\hline & 512 & $3.60 \mathrm{E}-07$ & 2.70 & $5.24 \mathrm{E}-05$ & 2.54 \\
\hline & 1,024 & $6.57 \mathrm{E}-08$ & 2.45 & $1.02 \mathrm{E}-05$ & 2.37 \\
\hline & 2,048 & $1.35 \mathrm{E}-08$ & 2.29 & $2.20 \mathrm{E}-06$ & 2.20 \\
\hline \multirow[t]{7}{*}{$p^{2}$} & 32 & $1.92 \mathrm{E}-04$ & - & $9.43 \mathrm{E}-03$ & - \\
\hline & 64 & $1.56 \mathrm{E}-05$ & 3.62 & $1.05 \mathrm{E}-03$ & 3.17 \\
\hline & 128 & $1.31 \mathrm{E}-06$ & 3.58 & $8.54 \mathrm{E}-05$ & 2.61 \\
\hline & 256 & $1.37 \mathrm{E}-07$ & 3.25 & $1.34 \mathrm{E}-05$ & 2.67 \\
\hline & 512 & $1.64 \mathrm{E}-08$ & 3.07 & $1.54 \mathrm{E}-06$ & 3.12 \\
\hline & 1,024 & $1.49 \mathrm{E}-09$ & 3.46 & $1.64 \mathrm{E}-07$ & 3.22 \\
\hline & 2,048 & $1.90 \mathrm{E}-10$ & 2.97 & $1.94 \mathrm{E}-08$ & 3.08 \\
\hline \multirow[t]{6}{*}{$p^{3}$} & 32 & $4.06 \mathrm{E}-05$ & - & $2.01 \mathrm{E}-03$ & - \\
\hline & 64 & $9.77 \mathrm{E}-07$ & 5.37 & $5.29 \mathrm{E}-05$ & 5.25 \\
\hline & 128 & $6.26 \mathrm{E}-08$ & 3.96 & $6.99 \mathrm{E}-06$ & 2.92 \\
\hline & 256 & $4.79 \mathrm{E}-09$ & 3.71 & $3.98 \mathrm{E}-07$ & 4.13 \\
\hline & 512 & $3.47 \mathrm{E}-10$ & 3.79 & $3.56 \mathrm{E}-08$ & 3.48 \\
\hline & 1,024 & $2.13 \mathrm{E}-11$ & 4.03 & $2.31 \mathrm{E}-09$ & 3.95 \\
\hline
\end{tabular}

In this example, we discuss the initial-boundary-value problem for the Eqs. (2.3a), (2.3b):

$\gamma(x, 0)=\gamma_{0}, \quad u(x, 0)=0$, for $0<x<L$, and $u(0, t)= \begin{cases}-V, & 0<t<T^{*}, \\ 0, & t>T^{*},\end{cases}$

where $T^{*}$ is the duration of the impact, $\gamma_{0}(\geq 0)$ is the constant pre-strain, and $V$ is the constant velocity of a sudden impact (we shall consider only tensile impact $V>0$ ). $\gamma_{0}=0$ means that the bar is assumed to be initially static in its undeformed state. Moreover, in order to satisfy the well-posedness of the equations, we impose nature boundary conditions at $x=0$. The boundary condition $u(0, t)$ generates an incoming impact from the left end $x=0$. We take $L$ to be large enough such that there is no disturbance at $x=L$ at the time of interest; that is, $u(t, L)=0, \gamma(t, L)=0$. 

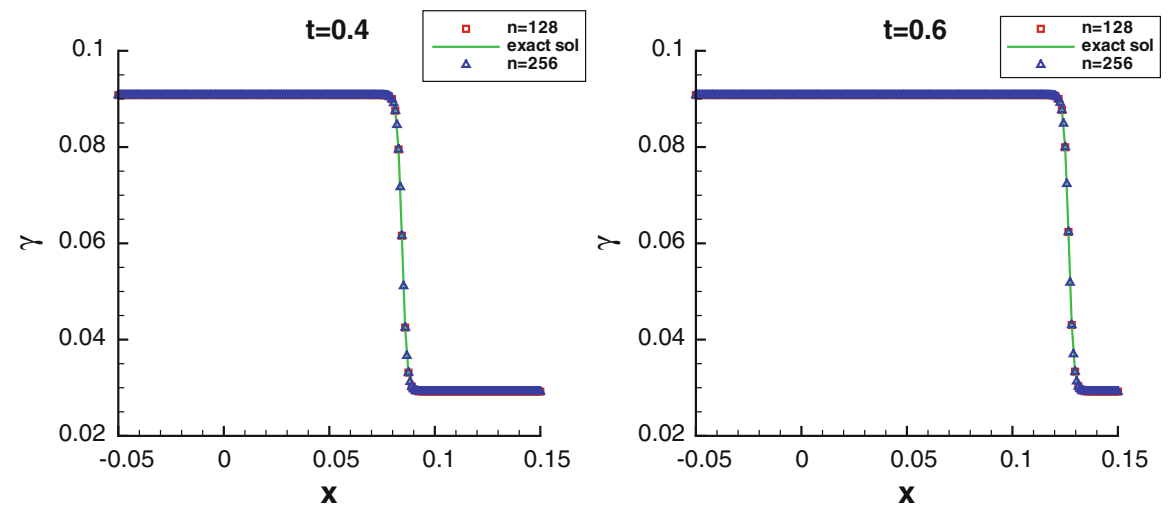

Fig. 2 The strain $\gamma$ for (5.1) at $t=0.4$ and 0.6 when $n=128,256$

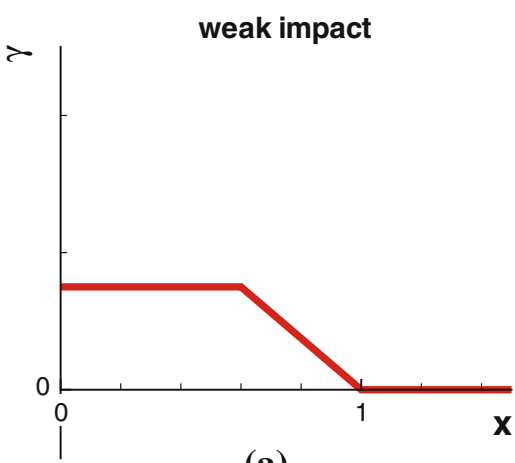

(a)

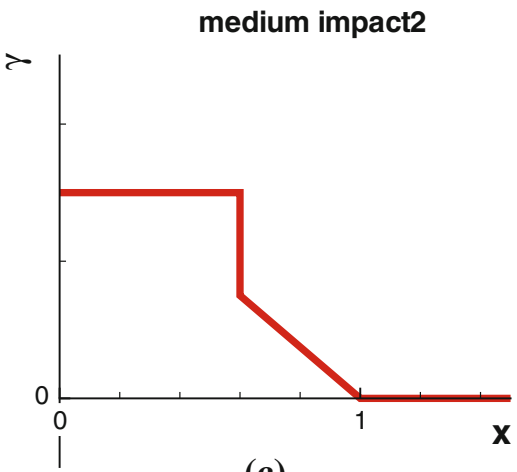

(c)

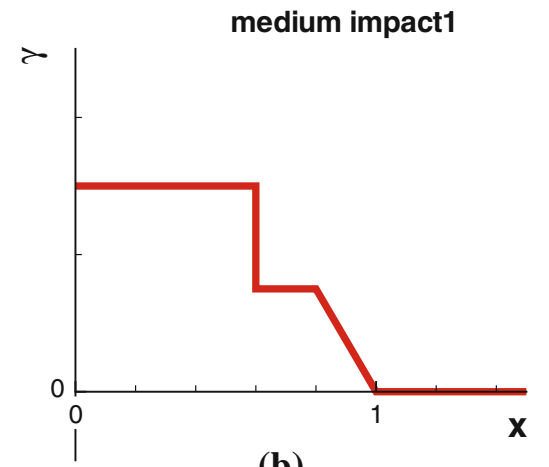

(b)

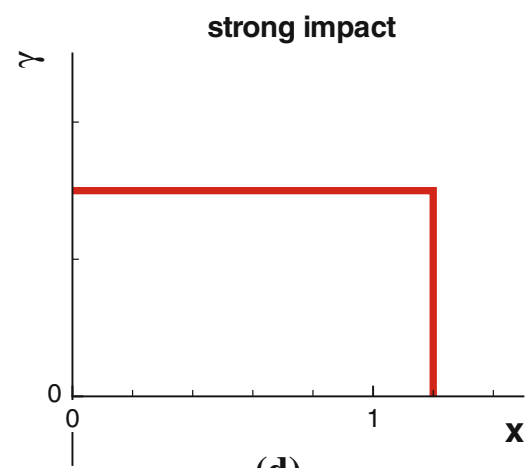

(d)

Fig. 3 The diagram for the strain $\gamma$ for various tensile impactor velocity $V$

In [9], Knowles described in detail the solutions in the three regimes of impactor velocity when $\gamma_{0}=0$, see Fig. 3. There is a pure fan response for a weak impact, there are a two-wave and a fan-plus-shock response for a medium impact (distinguishing 
by dissipation) and there is pure shock-wave response for a strong impact. In the following computation, we mainly compute the cases in Fig. 3(b) and (d).

In our computation, a uniformed mesh in $[0,2]$ is used for the LDG scheme, the computational time $t$ is to ensure that the waves do not propagate to the right boundary. Moreover, the duration of the impacts is $T^{*}=1.5$.

- The "analytical" solution for transformation front.

It is known that for the Eq. (1.1), a moving strain discontinuity at $x=s(t)$, the jump conditions can be written as

$$
\begin{gathered}
\dot{s}[\rho u]+[\sigma]=0, \\
\dot{s}[\gamma]+[u]=0,
\end{gathered}
$$

where $\dot{s}$ is the velocity of the moving discontinuity.

Moreover, according to Dai [6], when $\sigma(\gamma)$ is non-convex, for a traveling wave solution with transformation front, it has the third relations between the two phases, which can be written as

$$
[W(\gamma)]=[\gamma \sigma]-\frac{\rho}{2} \dot{s}^{2}\left[\gamma^{2}\right]
$$

Therefore, after some algebraic manipulation for three conditions (5.3)-(5.5), and considering the behavior of the transformation front, we can have

$$
\begin{gathered}
\gamma^{+}+\gamma^{-}=-\frac{2 D_{1}}{3 D_{2}}, \\
\dot{s}=\sqrt{\frac{\sigma\left(\gamma^{+}\right)-\sigma\left(\gamma^{-}\right)}{\gamma^{+}-\gamma^{-}},} \\
u^{+}+V-\sqrt{\left(\sigma\left(\gamma^{+}\right)-\sigma\left(\gamma^{-}\right)\right)\left(\gamma^{+}-\gamma^{-}\right)}=0, \\
u^{+}=-\int_{0}^{\gamma^{+}} \sqrt{\sigma^{\prime}(\gamma)} d \gamma .
\end{gathered}
$$

Together with (5.6)-(5.9), we can get $\gamma^{-}, \gamma^{+}$and $\dot{s}$ for the transformation front.

- The influence of the parameter $a$ (see Fig. 4).

We consider the convergence of the solution when the mesh is refined. We take $a=0.0006$ and $a=0.001$; for example, see Fig. 4. We find that when the mesh is refined, the solution when $a=0.001$ converges more quickly than when $a=0.0006$.

Remark 1 Here and in the sequel, we choose $a=0.001$ for our numerical computation.

- The relation between the velocity of transformation front $\dot{s}$ and the pre-strain $\gamma_{0}$ and the impact strength $V$ (see Figs. 5, 6). 

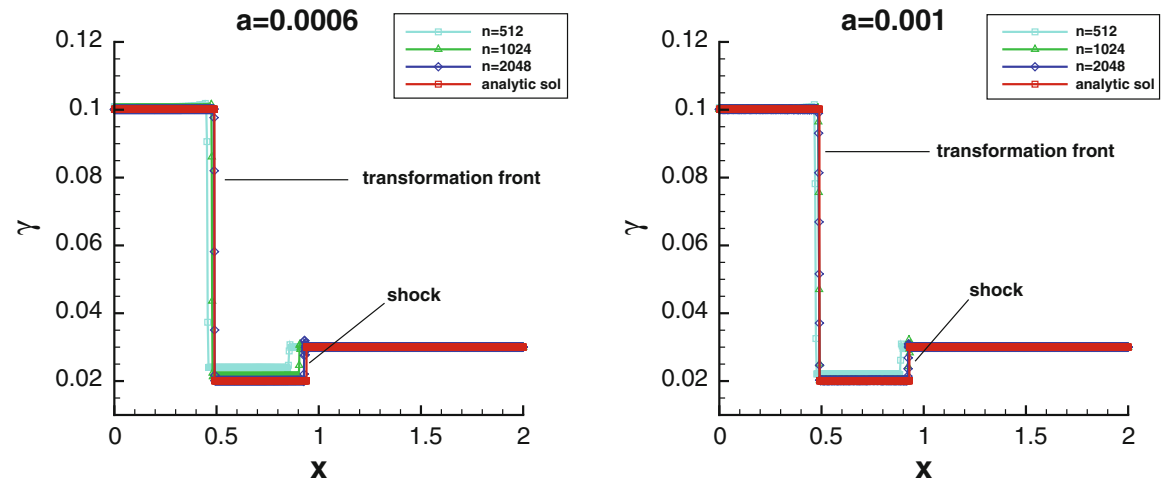

Fig. 4 The strain $\gamma$ with the parameter $\gamma_{0}=0.03, V=0.03, P^{1}$ element, $t=1.0$

Fig. 5 The strain $\gamma$ with the parameter $\gamma_{0}=0, P^{1}$ element, $n=4,096, t=1.0$

Fig. 6 The relation between the pre-strain $\gamma_{0}$ and the speed of transformation front $\dot{s}$ for different impacts
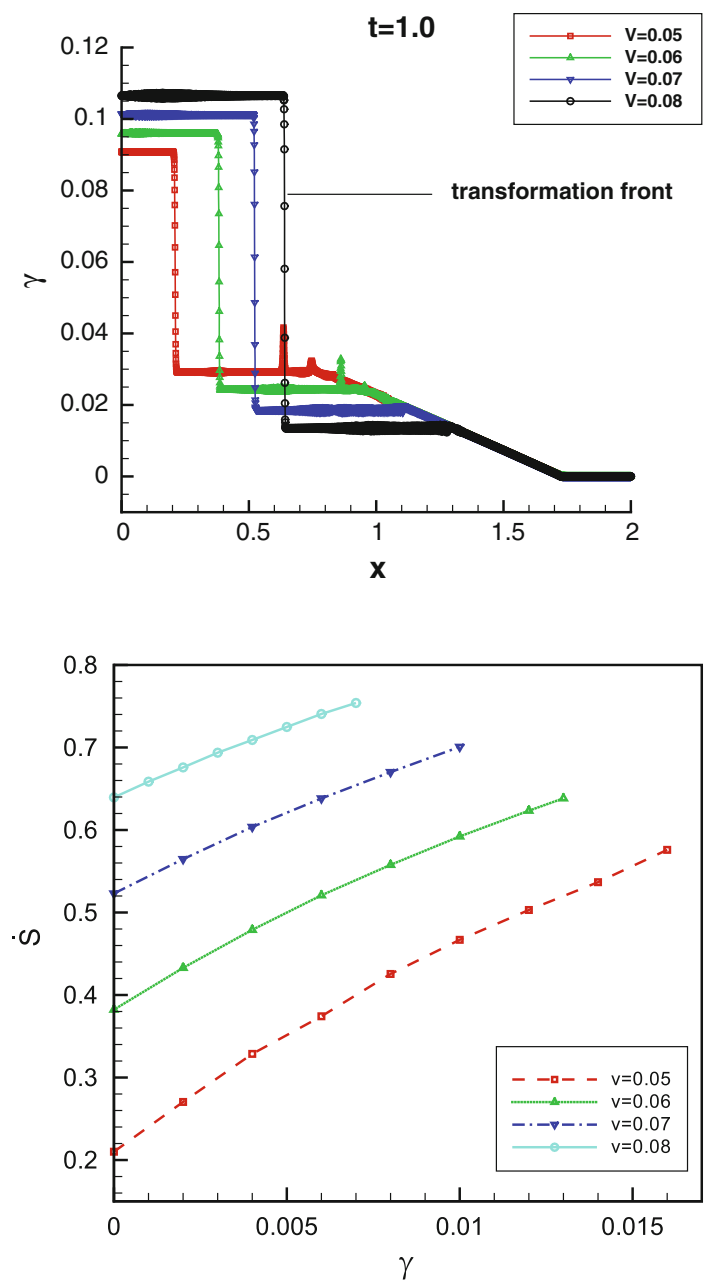

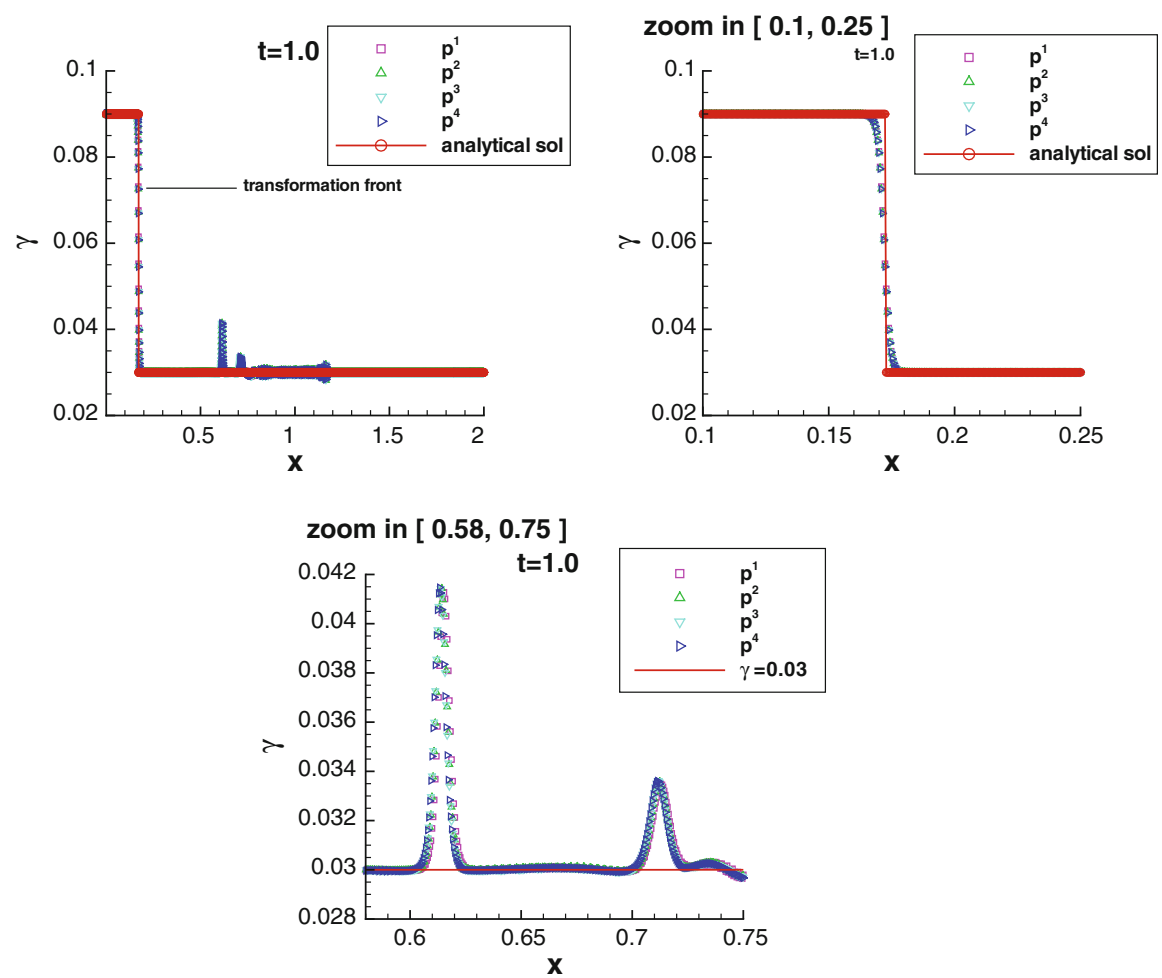

Fig. 7 The strain $\gamma$ with $\gamma_{0}=0.03, V=0.0103923, P^{k}, k=1,2,3,4$ element, $n=4,096, t=1.0$

We investigate the relation between the velocity of transformation front $\dot{s}$ and the pre-strain $\gamma_{0}$ and the impact strength $V$, see Figs. 5 and 6. From Fig. 5, We can see that the transformation front moves more quickly with increasing $V$. Moreover, from Fig. 6 , the velocity of transformation front becomes more and more large with $\gamma_{0}$ becoming large. We can conclude that the larger the parameters $\gamma_{0}$ and $V$, the larger the velocity $\dot{s}$ (i.e., the more quickly the transformation front moves). Note that in Fig. 5, when $V$ is relatively small, some soliton-like waves occur.

- Wave patterns with different pre-strains and different impacts (see Figs. 7-11).

According to Dai [7], we list some typical cases to present some interesting wave patterns.

1. $\gamma_{0}=0.03, V=0.0103923$; see Figs. 7 and 8 .

2. $\gamma_{0}=0.0267979, V=0.02$; see Figs. 9 and 10 .

3. $\gamma_{0}=0.02, V=0.02$; see Figs. 11 and 12 .

For the above three cases, we give comparing figures of the solution $\gamma$ using $P^{k}, k=$ 1, 2, 3, 4 element when $n=4,096$, and show the zoom-in figures (see Figs. 7, 9, 11); we also show the zoom-in figures when the mesh is refined (see Figs. 8, 10, 12). Moreover, from the zoom-in figures, we find that the numerical solution and the analytic solution for transformation agree with each other. 

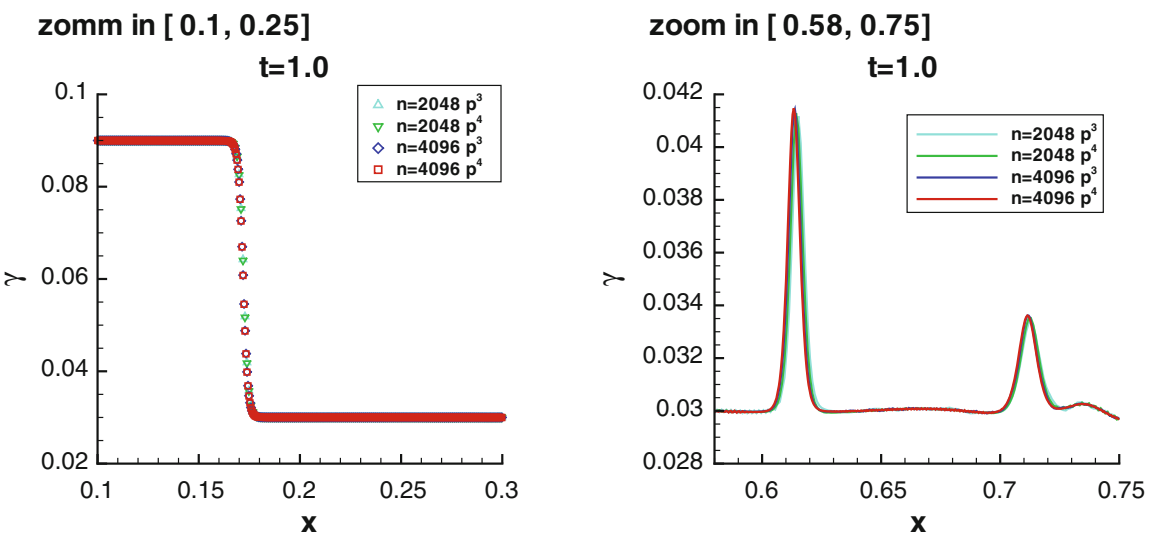

Fig. 8 The strain $\gamma$ with $\gamma_{0}=0.03, V=0.0103923, P^{k}, k=3,4$ element, $n=2,048,4,096, t=1.0$

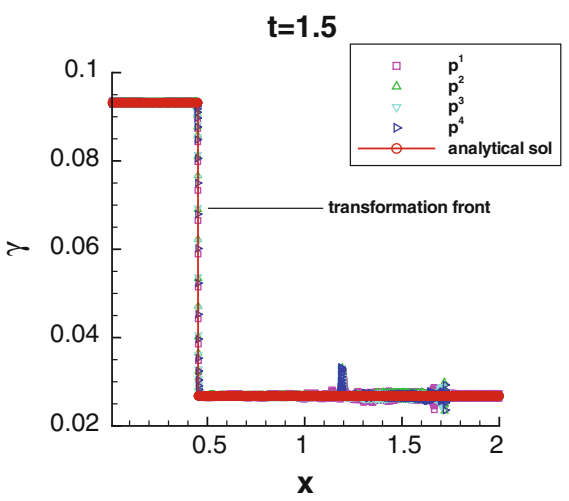

\section{zoom in [ 0.4, 0.55]}

$t=1.5$

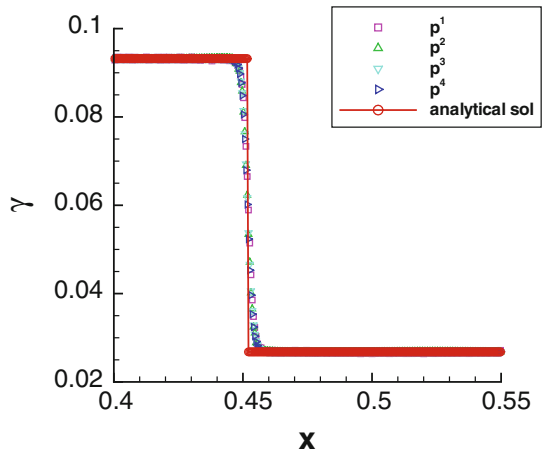

zoom in $[1.15,1.3]$

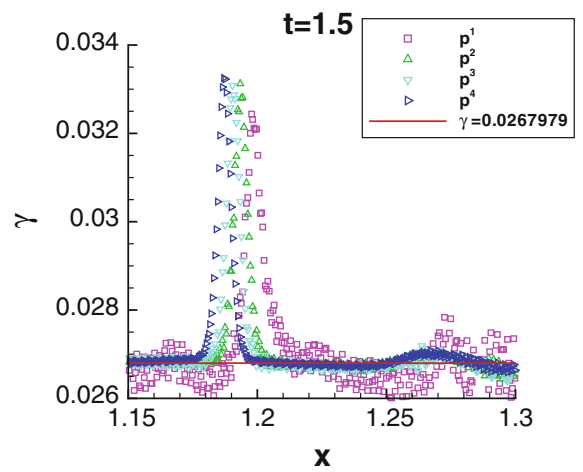

Fig. 9 The strain $\gamma$ with $\gamma_{0}=0.0267979, V=0.02, P^{k}, k=1,2,3$, 4 element, $n=4,096, t=1.5$

From Fig. 7, we find that, comparing with Fig. 4, for the same pre-strain $\gamma_{0}=0.03$, when impact $V$ changes from 0.03 to 0.0103923 , some soliton-like waves arise while the shock wave disappears. Moreover, the numerical solution and the analytic solution 
zoom in $[0.4,0.55]$

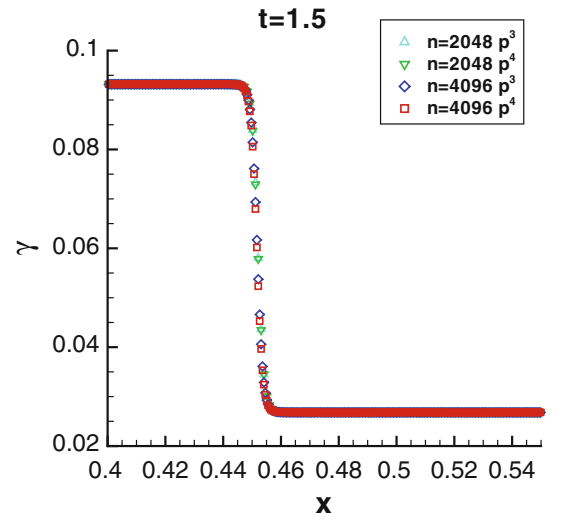

zoom in $[1.15,1.3]$

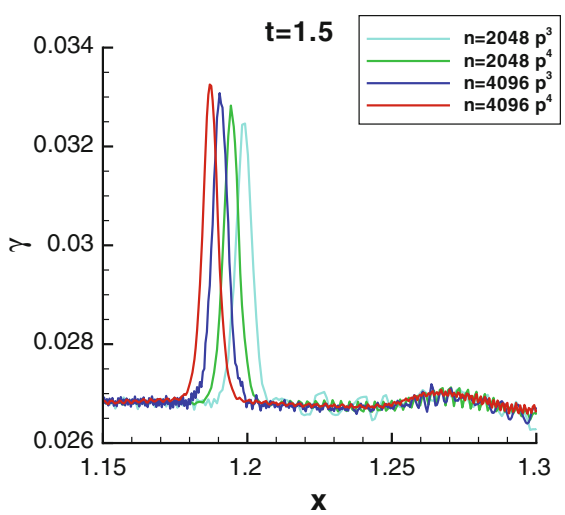

Fig. 10 The strain $\gamma$ with $\gamma_{0}=0.0267979, V=0.02, P^{k}, k=3,4$ element, $n=2,048,4,096, t=1.5$

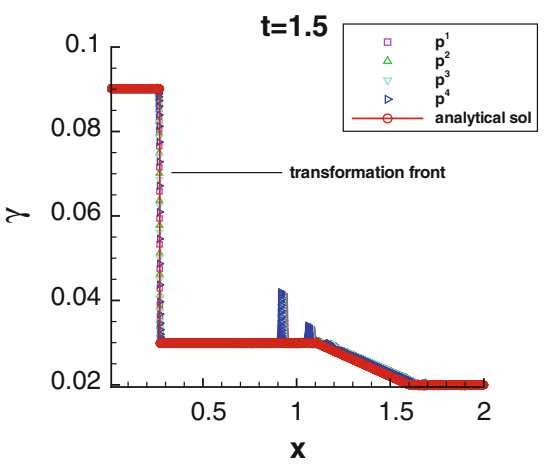

zoom in [ 0.2, 0.35]
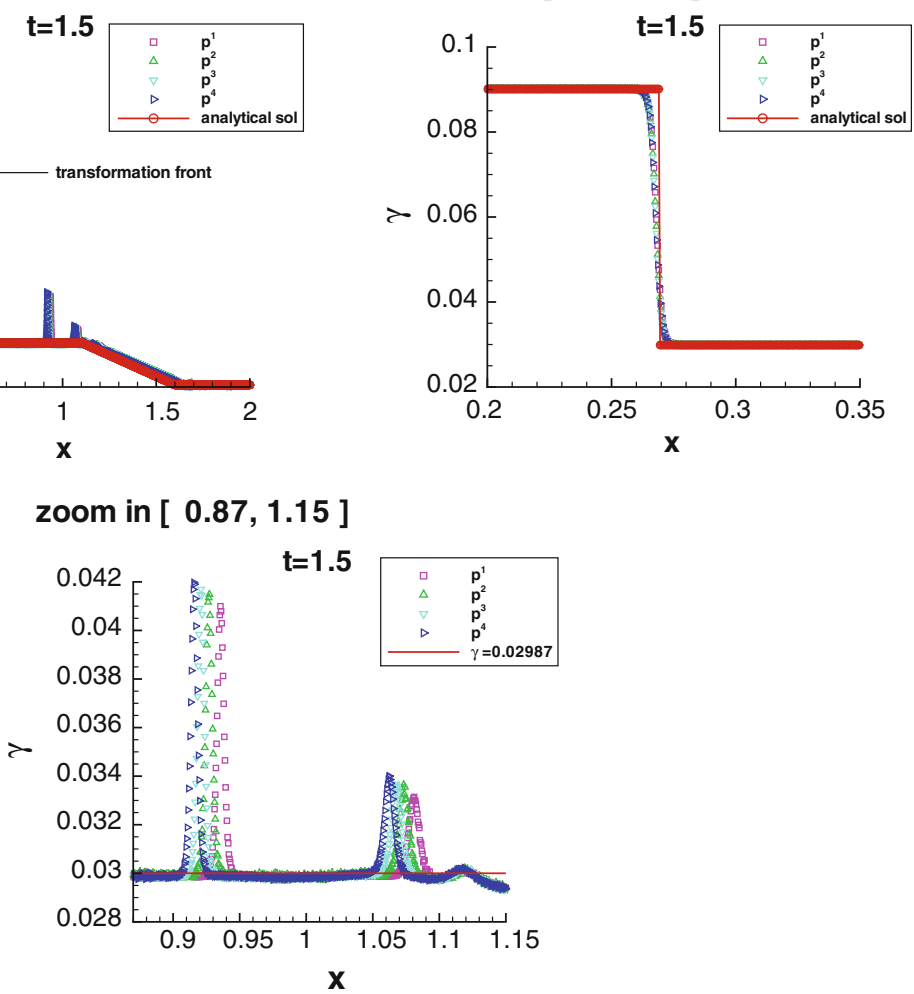

Fig. 11 The strain $\gamma$ with $\gamma_{0}=0.02, V=0.02, P^{k}, k=1,2,3,4$ element, $n=4,096, t=1.5$

for transformation agree with each other. There are few reports about this phenomenon of a solitary wave and a transformation front coexisting. 

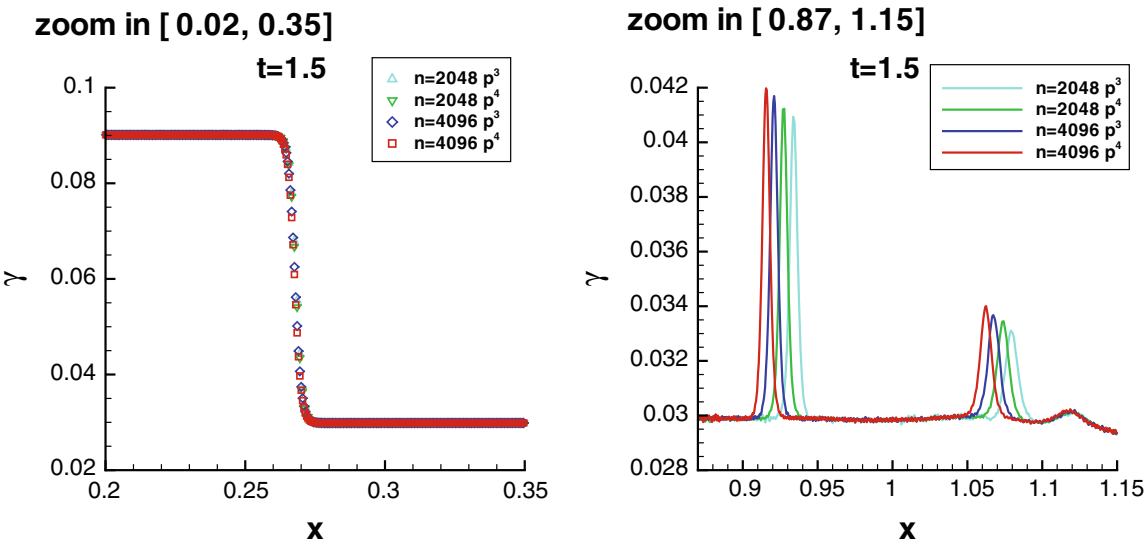

Fig. 12 The strain $\gamma$ with $\gamma_{0}=0.02, V=0.02, P^{k}, k=3,4$ element, $n=2,048,4,096, t=1.5$

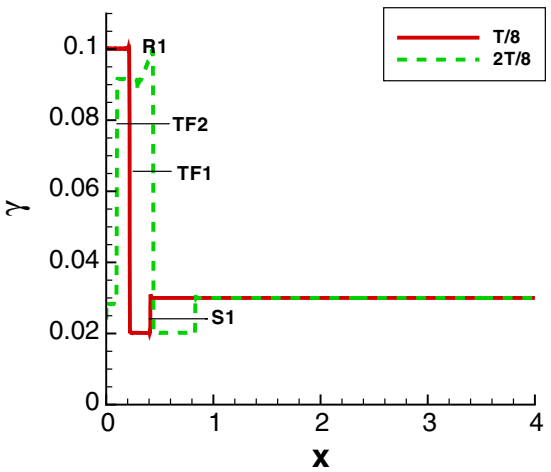

(a)

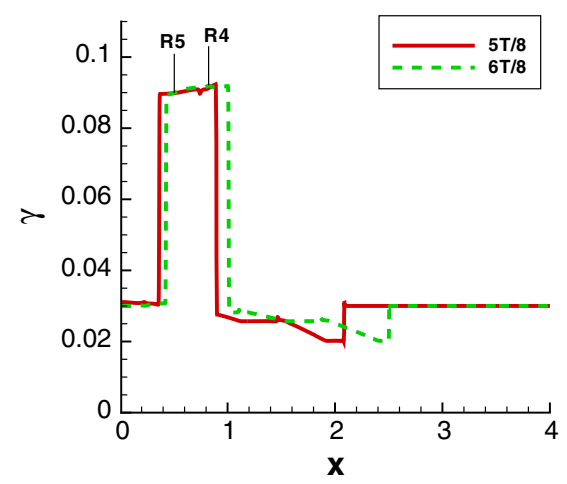

(c)

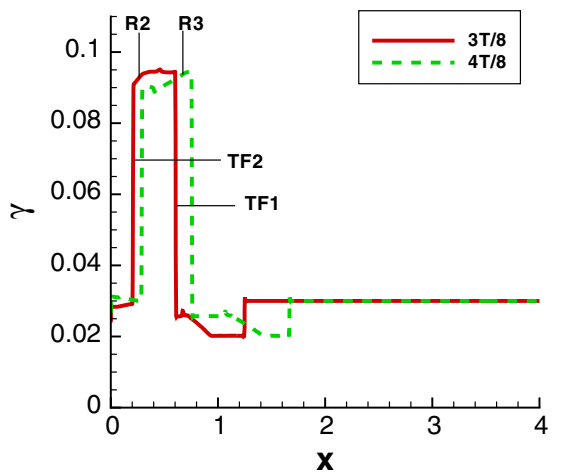

(b)

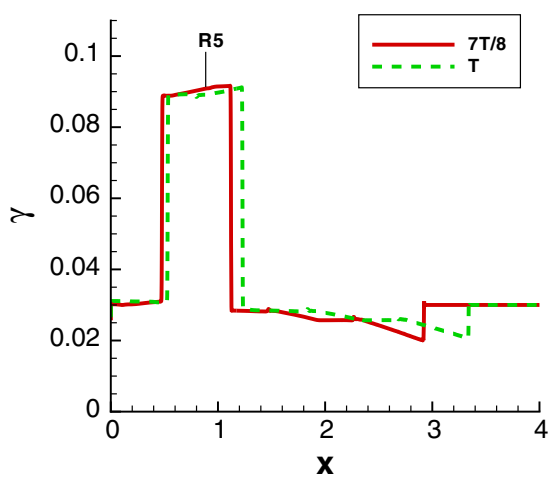

(d)

Fig. 13 The strain $\gamma$ for $\gamma_{0}=0.03, V=0.03$ at $t=T / 8,2 T / 8, \ldots, T, P^{1}$ element, $n=4,096, T=$ $3.6,[0,4]$ 
Fig. 14 The strain $\gamma$ for $\gamma_{0}=0.03, V=0.03$ at $t=1.5 T, 2 T, P^{1}$ element, $n=8,192,[0,8]$

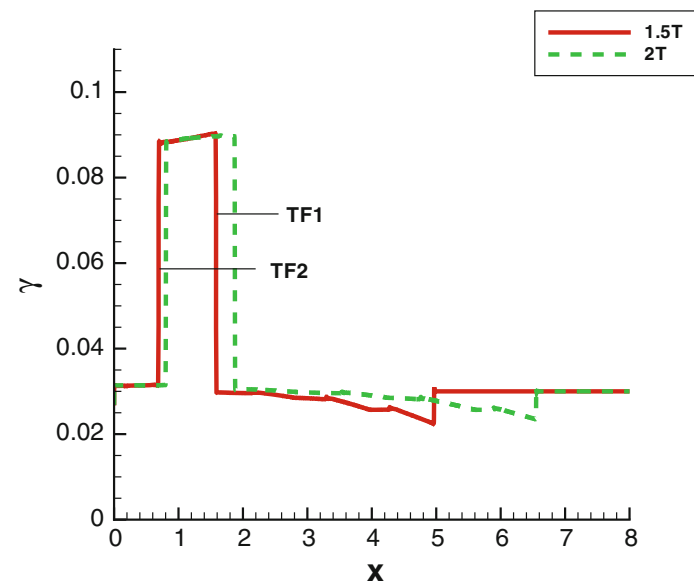

From Figs. 9 and 11, we can see that for the same impact $V=0.02$, there is only a transformation front when $\gamma_{0}=0.0267979$; when $\gamma_{0}$ becomes relatively small, a rarefaction wave occurs. More interestingly, soliton-like waves are formed for both the cases. Moreover, from the zoom-in figures, we find that there is a small difference at the solitary wave; when using the high-order element and refined mesh, the solitary waves relatively lag behind. In this paper, we mainly study the new wave patterns, thus we omit these details.

In fact, there are many situations according to $\gamma_{0}$, and the above two cases are typical of the interesting wave patterns.

- The interactions of rarefaction waves and transformation fronts (see Figs. 13, 14).

We will give the details of the interactions of rarefaction waves and transformation fronts when $\gamma_{0}=0.03, V=0.03$. The computational interval is $[0,4]$, the duration of the impacts is $T^{*}=0.5$. We take $n=4,096, T=3.6$. The numerical simulation for $t=\frac{T}{8}, \frac{2 T}{8}, \ldots, T$ is shown in Fig. 13 .

From Fig. 13(a), when $t \leq 0.5$, the wave pattern is a right-going transformation front (TF1) and a right-going shock wave ( $S 1)$. When $t \geq 0.5$, a new transformation front (TF2) and a right-going rarefaction wave $(R 1)$ are generated. From Fig. 13(b), we find that $R 1$ propagates faster than the TF1 to the right, then it interacts with TF1, and the magnitude changes from 0.0997 to 0.0951 . Meanwhile, a new smaller rarefaction wave $(R 2)$ is formed in the left, it will interact with TF2. Then a new rightgoing rarefaction wave (TF3) is created, which will interact with TF1. As the time evolves, the above process is repeated, and new rarefaction waves like $R 4$ and $R 5$ are generated (see Fig. 13(c), (d)). During the processes of interaction, rarefaction waves are formed one by one until there is a small difference with the TF1 and TF2.

From Fig. 14, we find that the TF1 and TF2 become more and more close with each other. 


\section{Concluding Remarks}

We use the LDG method to solve a mixed-type hyperbolic-elliptic system, in a slender cylinder, which includes material nonlinearity (due to the non-monotone of strainstress function) and dispersion terms (due to the effects of the geometrical size of the cylinder). We give the stability for the LDG scheme. In order to verify the validity of the LDG scheme, we give the errors and the accuracy of a numerical example. Due to the interaction of the dispersion and the material nonlinearity, some interesting wave patterns occur; such as the pattern with transformation front and solitary wave, the pattern with rarefaction wave and solitary wave. We also investigate the interaction of transformation fronts and rarefaction waves. It is worthy to be mentioned that the model Eq. (2.1) introduced in Dai [6] is subject to criticism since the dissipation effect is not taken into account (which is important in a phase transition). The model (2.1) of Dai [6], although one-dimensional, takes into account some three-dimensional effects. We can consider to add a viscoelastic term $\left(\mu u_{x x t}\right.$, where $\mu$ is the viscosity coefficient) for dissipation to the Eq. (2.1), which has been used by some authors (see $[8,17,18])$. Then, the resulting model is more physically sound and it is worthwhile to do numerical investigations.

Acknowledgments Research supported by NSFC Grant Nos. 11371342, 11031007, FANEDD No. 200916, Fok Ying Tung Education Foundation No. 131003.

\section{References}

1. Abeyaratne, R., Knowles, J.K.: Kinetic relations and the propagation of phase boundary in solids. Arch. Ration. Mech. Anal. 114, 119-154 (1991)

2. Abeyaratne, R., Knowles, J.K.: Evolution of Phase Transitions: A Continuum Theory. Cambridge University Press, Cambridge (2006)

3. Cockburn, B., Gau, H.: A model numerical scheme for the propagation of phase transitions in solids. SIAM J. Sci. Comput. 17, 1092-1121 (1996)

4. Cockburn, B., Shu, C.-W.: The local discontinuous Galerkin method for time-dependent convectiondiffusion systems. SIAM J. Numer. Anal. 35, 2440-2463 (1998)

5. Cockburn, B., Shu, C.-W.: The Runge-Kutta discontinuous Galerkin methods for convectiondomainated problems. J. Sci. Comput. 16, 173-261 (2001)

6. Dai, H.H.: Non-existence of one-dimensional stress problems in solid-solid phase transitions and uniqueness conditions for incompressible phase-transforming materials. C. R. Acad. Sci., Paris, Ser. I 338, 981-984 (2004)

7. Dai, H.H., Xu, Z.L.: Impact-induced wave patterns in a slender cylinder composed of a non-convex elastic material. AIP Conf. Proc. 1029, 77 (2008). doi:10.1063/1.2971996

8. Făciu, C., Mihăilescu-Suliciu, M.: On modelling phase propagation in SMAs by a Maxwellian thermoviscoelastic approach. Int. J. Solids Struct. 39, 3811-3830 (2002)

9. Knowles, J.K.: Impact-induced tensile waves in a rubberlike material. SIAM J. Appl. Math. 62, 11531175 (2002)

10. Li, Z.Q., Sun, Q.P.: The initiation and growth of macroscopic martensite band in nano-grained NiTi microtube under tension. Int. J. Plast. 18, 1481-1498 (2002)

11. Shaw, J.A., Kyriakides, S.: Thermomechanical aspects of NiTi. J. Mech. Phys. Solids 47, 1243-1281 (1995)

12. Shaw, J.A., Kyriakides, S.: On the nucleation and propagation of phase transition fronts in a NiTi alloy. Acta Mater. 45, 683-700 (1997)

13. Shaw, J.A., Kyriakides, S.: Initiation and propagation of localized deformation in elasto-plastic strips under uniaxial tension. Int. J. Plast. 13, 837-871 (1998) 
14. Shu, C.-W., Osher, S.: Efficient implementation of essentially non-oscillatory shock capturing schemes. J. Comput. Phys. 77, 439-471 (1988)

15. Slemrod, M.: Dynamic phase transitions in a van der Waals fluid. J. Differ. Equ. 52, 1-23 (1984)

16. Tian, L.L., Xu, Y., Kuerten, J.G.M., Van der Vegt, J.J.W.: A local discontinuous Galerkin method for the propagation of phase transition in solids and fluids. J. Sci. Comput. (2013). doi:10.1007/ s10915-013-9778-9

17. Vainchtein, A.: Dynamics of non-isothermal martensitic phase trasitions and hysteresis. Int. J. Solids Struct. 39, 3387-3408 (2002)

18. Vainchtein, A.: Dynamics of phase transitions and hysteresis in a viscoelastic Ericksen's bar on an elastic foundation. J. Elast. 57, 243-280 (1999)

19. Xu, Y., Shu, C.-W.: Local discontinuous Galerkin methods for three classes of nonlinear wave equations. J. Comput. Math. 22, 250-274 (2004)

20. Xu, Y., Shu, C.-W.: Local discontinuous Galerkin methods for nonlinear Schrö dinger equations. J. Comput. Phys. 205, 72-97 (2005)

21. Xu, Y., Shu, C.-W.: Local discontinuous Galerkin methods for two classes of two dimensional nonlinear wave equations. Physica D 208, 21-58 (2005)

22. Xu, Y., Shu, C.-W.: Local discontinuous Galerkin methods for the Kuramoto-Sivashinsky equations and the Ito-type coupled KdV equations. Comput. Methods Appl. Mech. Eng. 195, 3430-3447 (2006)

23. Xu, Y., Shu, C.-W.: Error estimates of the semi-discrete local discontinuous Galerkin method for nonlinear convection-diffusion and KdV equations. Comput. Methods Appl. Mech. Eng. 196, 38053822 (2007)

24. Xu, Y., Shu, C.-W.: A local discontinuous Galerkin methods for the Camassa-Holm equations. SIAM J. Numer. Anal. 46, 1998-2021 (2008)

25. Yan, J., Shu, C.-W.: A local discontinuous Galerkin method for KdV type equations. SIAM J. Numer. Anal. 40, 769-791 (2002)

26. Yan, J., Shu, C.-W.: Local discontinuous Galerkin methods for partial differential equations with higher order derivatives. J. Sci. Comput. 17, 27-47 (2002) 